Pueblos originarios y Ley de Servicios de Comunicación Audiovisual. El caso Wall Kintun TV

María Cecilia Hang

Tram[p]as de la comunicación y la cultura (N. $\left.{ }^{\circ} 84\right)$, e036, 2019

ISSN 2314-274X | https://doi.org/10.24215/2314274xe036

http://perio.unlp.edu.ar/ojs/index.php/trampas

FPyCS | Universidad Nacional de La Plata

La Plata | Buenos Aires | Argentina

\title{
PUEBLOS ORIGINARIOS Y LEY DE SERVICIOS DE COMUNICACIÓN AUDIOVISUAL
}

\author{
EL CASO WALL KINTUN TV \\ INDIGENOUS PEOPLES AND THE AUDIOVISUAL COMMUNICATION \\ SERVICES ACT: THE WALL KINTUN TV CASE \\ Maria Cecilia Hang \\ mariaceciliahang@gmail.com \\ https://orcid.org/0000-0003-4957-9431 \\ Consejo Nacional de Investigaciones Científicas y Técnicas (CONICET) \\ Universidad Nacional de San Luis | Argentina
}

\begin{abstract}
El trabajo aborda el reconocimiento del derecho a la identidad de los pueblos originarios en su rol de prestadores de servicios de comunicación audiovisual a partir de analizar el caso de Wall Kintun TV, el primer canal de televisión mapuche, en el contexto de la creación y la implementación de la Ley de Servicios de Comunicación Audiovisual (LSCA) como resultado del proceso de recuperación de la tradición crítica latinoamericana.
\end{abstract}

\begin{abstract}
The paper analyzes the recognition of indigenous peoples' right to identity in their role as audiovisual communication services' providers from analyzing the case of Wall Kintun TV, the first mapuche television channel, in the context of the creation and introduction of the Audiovisual Communication Services Act (LSCA) as a result of the process of recovery of the Latin American critical tradition.
\end{abstract}

Palabras clave | Ley de Servicios de Comunicación Audiovisual, pueblos originarios, Wall Kintun TV, política pública

Keywords | Audiovisual Communication Services Act, indigenous peoples, Wall Kintun TV, public policies

Recibido: 31/07/2019 | Aceptado: 05/10/2019 | Publicado 20/11/2019 


\title{
PUEBLOS ORIGINARIOS Y LEY DE SERVICIOS DE COMUNICACIÓN AUDIOVISUAL
}

\author{
EL CASO WALL KINTUN TV
}

Por Maria Cecilia Hang

¡Marici wev! ¡Marici wev! ¡Diez veces estamos vivos! ¡Diez veces venceremos! (Deolinda del Pilar Buenuleo, 2012)

Breve génesis de la LSCA

La Ley de Servicios de Comunicación Audiovisual (LSCA), sancionada en 2009, reemplazó a su antecesora que fuera heredada de gobiernos dictatoriales. El proyecto de la nueva y mal llamada "ley de medios" (porque solo regula los servicios de radiofonía y TV) comenzó a gestarse en 2004 cuando la Coalición por una Radiodifusión Democrática, integrada por organizaciones sindicales de trabajadores, movimientos sociales, partidos y organizaciones politicas, universidades nacionales, cooperativas, radios y canales comunitarios, PYMES y los pueblos originarios, elaboró un documento con los 21 puntos básicos por el derecho a la comunicación. Este concepto merece una atención especial ya que, adscribiendo a las normativas internacionales, se reivindica como:

La comunicación es un derecho fundamental e irrenunciable, que no pasa exclusivamente por la tenencia de los medios pero que tiene que contemplar este capitulo, y que se apoya fundamentalmente en los criterios de acceso y participación en igualdad de oportunidades para ingresar en la agenda temática de la sociedad, en la producción de mensajes, en el intercambio de ideas, en el debate (Coalición por una Radiodifusión Democrática, 2004, p. 3). 
Este fragmento implica pensar a las ciudadanas y a los ciudadanos no como meros receptores de la información sino como sujetos activos, capaces de producir y de emitir contenidos. En este sentido, los pueblos originarios intervinieron en la construcción del documento y reclamaron la inclusión de la comunicación como derecho a la identidad. Esto implica el reconocimiento de su capacidad para elaborar estrategias comunicacionales propias (Manasanch, 2010). Según relata Néstor Piccone, cofundador de la Coalición por una Comunicación Democrática e integrante del Consejo Asesor de Televisión Digital Abierta, la inclusión de los reclamos de los pueblos originarios se realizaron sobre la base de la categoría jurídica reconocida en la reforma constitucional de 1994, que implica considerarlos como pueblos dentro del Estado Nacional, con identidad propia y con derechos colectivos que se derivan de su presencia histórica y contemporánea (Visión Indígena, 14/12/2012, en linea).

En cuanto al contexto político y social latinoamericano en el marco del cual se sancionó la LSCA, la presencia de gobiernos de izquierda o progresistas ${ }^{1}$ volvió a colocar en la agenda pública la discusión sobre las políticas de comunicación y la importancia de la intervención del Estado sobre los sistemas de medios heredados del régimen neoliberal. Este debate resultó en la construcción de proyectos de normativas o en la promulgación de leyes sobre políticas públicas de comunicación, producto de la recuperación de la tradición crítica iniciada en la década del setenta.

\section{La LSCA y la recuperación}

de la tradición critica latinoamericana

A partir de la década del setenta surge la tradición crítica latinoamericana, que se extiende hasta la elaboración del Informe MacBride, en 1980 (Tremblay, 2005). Durante este periodo se produjeron una gran cantidad de trabajos científicos en los que la comunicación era pensada desde contextos latinoamericanos y necesidades locales. La preocupación central radicaba en la denuncia de los efectos ideológicos de los mensajes teniendo en cuenta que los desequilibrios comunicacionales (lo urbano y lo rural, clases bajas y altas, subordinación a los contenidos estadounidenses) resultaban en productos culturales que solo recuperaban los intereses hegemónicos. Es así que se 
advirtió sobre la necesidad de construir políticas nacionales de comunicación que intervinieran sobre la concentración de los medios, aunque, según Martín Becerra y Guillermo Mastrini (2009), esta perspectiva olvidó la dimensión económica de la producción cultural.

Este debate se desarrolló en paralelo con el que mantenían los países "no alineados" en el seno de la Organización de las Naciones Unidas (ONU) en torno al Nuevo Orden Económico Internacional (NOEI) y el Nuevo Orden Mundial de la Información y la Comunicación (NOMIC). De este último grupo surgió la Comisión Internacional para el Estudio de los Problemas de Comunicación que en 1980 presentó el famoso Informe MacBride, una caja de resonancia de los debates latinoamericanos. En el documento se advirtió sobre la importancia de la democratización de las comunicaciones en el orden mundial y se denunció el desequilibrio en los flujos informacionales y la necesidad de garantizar el acceso a mejoras tecnológicas de los países más atrasados en materia de equipos de información y de comunicación.

Sin embargo, estos esfuerzos se realizaron en un contexto que no permitió su materialización en decisiones concretas sobre el sistema de medios. La ideología hegemónica de la época propuso como solución la liberalización del mercado. La salida de Gran Bretaña y de Estados Unidos de la UNESCO debilitó y deslegitimó el debate internacional por un orden comunicacional más justo y equitativo.

En la década del ochenta, la actitud "denuncista" fue reemplazada por sistemas neoliberales que cuestionaron el papel interventor del Estado debido a que lo considerban restrictivo de la circulación de bienes culturales. Esto dio como resultado la falta de control$^{2}$ sobre el sistema informacional, el cual presentaba las siguientes características:

- Privatización de medios de comunicación.

- Ingreso de capitales extranjeros a las empresas del sistema de medios nacionales, lo que favoreció la entrada de grupos transnacionales en las industrias culturales latinoamericanas.

- Conformación de grandes corporaciones mediáticas nacionales y transnacionales. 
- Centralización urbana de contenidos.

- Ausencia de medios públicos.

Los gobiernos latinoamericanos no intervinieron en las politicas de comunicación e ignoraron la normativa, lo que permitió que las decisiones fueran permeables a los intereses de las grandes corporaciones de multimedios.

En este contexto llegaron al poder los gobiernos de izquierda o progresistas que reactivaron la legitimidad del papel del Estado para intervenir en la economía y en la sociedad, por lo que se destinaron esfuerzos a la elaboración y a la implementación de políticas públicas de comunicación. Al respecto, se expresa que "aunque el consenso de las fuerzas sociales en torno a ellas está lejos de ser conseguido, el debate que vuelve a plantearse después de largos años de voces únicas en la región es en cualquier caso enriquecedor" (Badillo, Mastrini \& Marenghi, 2015, p. 108).

\section{La LSCA y la mirada sobre los medios \\ de comunicación como servicio de interés público}

La LSCA recupera en su texto la preocupación de la tradición latinoamericana sobre la concentración de la propiedad y la necesidad de la intervención estatal en los sistemas de medios; en este sentido, se retoman los conceptos de acceso, universalidad, desconcentración e interés público, entre otros. En consecuencia, los medios de comunicación son concebidos como un servicio de interés público y se postulan algunas ideas de la tradición europea, entre las que podemos destacar:

- Intervención del Estado en el sistema de medios que garantice la prestación de un servicio que es considerado esencial.

- Función social y no exclusivamente económica: entretenimiento, información y educación de alta calidad. Los medios son clave para la construcción de un sistema democrático que represente a una multiplicidad de identidades y de intereses sociales. Son formadores de la subjetividad social y de opinión pública. 
- Diversidad de contenidos: no es solo la búsqueda de la ganancia sino la prestación de un servicio que abarque los contenidos y los gustos de una pluralidad de grupos y no únicamente de aquellos que son económicamente más rentables.

- Universalidad: disponibilidad del servicio para todos en igualdad de condiciones, sin distinción de localización geográfica.

- Acceso a bienes culturales y fuentes de información plurales.

- Participación de la ciudadanía como sujeto de la política y no solo como objeto.

- Prestadores de gestión estatal, de gestión privada con fines de lucro y de gestión privada sin fines de lucro. En este sentido, se establece la intervención del Estado para controlar inversiones, tarifas y para asegurar el acceso y la participación en igualdad de condiciones.

\section{Nuevo paradigma legal latinoamericano}

Como señalan Daniel Badenes y Néstor González (2014), el reconocimiento del sector privado sin fines de lucro o comunitario como prestador específico es un aspecto destacable del nuevo paradigma legal latinoamericano, fruto de la lucha por la democracia comunicacional sostenida por las organizaciones sociales, que generó las condiciones de acceso para nuevos actores al campo de los servicios de comunicación audiovisual.

No obstante, este paradigma se encuentra con las tensiones planteadas por la multietnicidad latinoamericana en el proceso de construcción de un "techo común». En este sentido Néstor García Canclini (2004), plantea:

Así como en la lengua tzeltal, en Chiapas, el término "derechos" de la Declaración Universal de los Derechos Humanos se traduce por la expresión ich ' el ta muk, o sea respeto, los pueblos necesitan ir y venir entre pedir que sus derechos sean reconocidos en el mundo moderno y tratar de, al menos, ser respetados en su mundo, que por supuesto no está fuera de la modernidad (p. 135). 
Estos procesos de idas y vueltas se vinculan con la cosmovisión mapuche sobre la comunicación, recuperada por Florencia Yanniello (2014) y por Gutiérrez Ríos (2014) y a la cual definen como "comunicación con identidad". Este concepto implica, para quienes integran las comunidades originarias, la generación de medios de comunicación propios para transmitir sus maneras de ver el mundo, es decir, difundir palabras y conceptos que representan su identidad y contrarrestar un discurso dominante.

El medio, entonces, ahora tiene un apellido: video mapuche, diario mapuche, radio mapuche. Esta apropiación se desarrolla a través de los medios que son considerados resignificables; "es decir, apropiar lo que de afuera sirve para fortalecer o acompañar el proceso y si, por el contrario, trae problemas y afecta el territorio se expulsa, pero queda la experiencia", como señala Vilma Almendra (en Gutiérrez Ríos, 2014, p. 43), a propósito de la experiencia del pueblo nasa en Colombia.

Se apropian discursos y medios de la cultura visual occidental para resignificarlos y para usarlos de una manera específica: ofrecer una voz divergente y crítica a la construcción de "lo mapuche" en la esfera pública tradicional. En este sentido, los medios están al servicio de la organización, del proceso y de la conciencia colectiva.

En consecuencia, y como se analizará en los próximos apartados, los pueblos originarios reclaman al Estado un reconocimiento que trasciende lo simbólico para exigir el cumplimiento de derechos concretos. El Estado es el «espacio de protección" en la figura de su autoridad y sus servicios, pero este proceso impone un limite: un reconocimiento de sus derechos hacia afuera y un dejar hacer hacia adentro. Se plantea una tensión entre lo hegemónico y lo particular que implica otro desafio para la normativa: cómo reconocer derechos sin avasallar identidades.

\section{La LSCA y los pueblos originarios}

Teniendo en cuenta lo desarrollado hasta el momento, en este apartado se especificarán los artículos de la LSCA que se refieren a los derechos reconocidos a los Pueblos Originarios como prestadores de servicios de comunicación audiovisual. 
En primer lugar, en el artículo 3 (Ley 26.522, 2009) se establece que uno de los objetivos de los servicios de comunicación audiovisual y de los contenidos de sus emisiones, es la preservación y la promoción de la identidad y de los valores culturales de los pueblos originarios.

La referida ley reconoce a los pueblos originarios como sujetos plenos de derecho a los cuales se les otorgan licencias de acuerdo con su demanda, de manera directa y de acuerdo a la disponibilidad del espectro. La petición de la licencia se realiza a través de la presentación de un proyecto ante la Autoridad Federal de Servicios de Comunicación Audiovisual; la asignación se realiza de manera directa, con el único requisito de la disponibilidad del espectro donde se pretenda poner en marcha el medio. Para ello, en el artículo 89 inc. e (Ley 26.522, 2009), se especifican las reservas del espectro radioeléctrico en las localidades donde cada pueblo se encuentre asentado (una frecuencia de AM, una de FM y una frecuencia de televisión).

La financiación se especifica en el artículo 97 inc. f (Ley 26.522, 2009), que establece que el diez por ciento de lo recaudado mediante gravámenes deberá ser destinado para "proyectos especiales de comunicación audiovisual y apoyo a servicios de comunicación audiovisual, comunitarios, de frontera, y de los pueblos originarios, con especial atención a la colaboración en los proyectos de digitalización". En 2013 comenzó a aplicarse con los dos llamados del Fondo de Fomento Concursable para Medios de Comunicación Audiovisual (FOMECA) cuyo mecanismo se encuentra previsto en el artículo 153 (Ley 26.522, 2009) y está destinado a organizaciones sin fines de lucro y de pueblos originarios que se encuentren legalizados, reconocidos o autorizados. Pero el artículo 97 también considera otro tipo de financiamiento como asignaciones del presupuesto nacional, venta de publicidad, donaciones, venta de contenidos de producción propia, auspicios o patrocinios y recursos específicos asignados por el Instituto Nacional de Asuntos Indígenas.

\section{¿Cómo se aplicó la normativa en el caso de Wall Kintun TV?}

En un estudio realizado por Santiago Marino, Guillermo Mastrini, Martín Becerra, Carolina Rubini y Agustín Espada (2015) se analizaron las autorizaciones de medios de comunicación otorgadas a pueblos originarios en el período comprendido entre 2011 y 2015. 
Se pudo constatar que se otorgaron 46 señales, de las cuales una pertenece a TV abierta, una a TV Digital y las restantes son estaciones de FM. La comunidad mapuche recibió la señal de TV abierta y Digital, además de otras 10 radios $\mathrm{FM}$, lo cual la posiciona como el pueblo originario con más autorizaciones de señales en vigencia.

En cuanto a la información de tipo cualitativa, fue complejo acceder a los datos oficiales ya que no se encuentran disponibles en la web. Es por eso que se realizó una búsqueda en la red, en donde se recabaron datos de portales de noticias y de blogs. El análisis no intenta ser exhaustivo sino un acercamiento preliminar al objeto de estudio.

El 7 de diciembre de 2012 los medios argentinos anunciaron la inauguración del primer canal de televisión de pueblos originarios, Wall Kintun TV, en la ciudad de Bariloche. Se informó que el canal se visualizaría en el aire del Canal 2 de la mencionada localidad y que la primera programación a emitir sería Visión Indigena, una producción de la Coordinadora de Comunicación Audiovisual Indígena Argentina que narraría la actualidad de las distintas comunidades del país (Premici, 08/12/2012). El canal estaría a cargo de la Cooperativa de Servicios Audiovisuales Mapuche, conformada por 15 comunicadores (Lorenzo, 2012), y la programación incluiría dos contenidos: uno sobre politica intercultural, con representantes de la politica y de los pueblos originarios, y otro con entrevistas a personalidades de la comunidad, con el objetivo de que puedan compartir sus historias de vida (Premici, 08/12/2012).

Según ambas noticias, la Autoridad Federal de Servicios de Comunicación Audiovisual (AFSCA), el Instituto Nacional de Asuntos Indígenas (INAI), la Comisión Nacional de Comunicaciones (CNC) y la Coordinadora de Comunicación Audiovisual Indígena capacitaron a más de 200 jóvenes.

Los promotores y las promotoras de esta iniciativa obtuvieron el equipamiento para producir y para transmitir con la ayuda de AFSCA y del Sistema Nacional de Televisión Digital Abierta (Lorenzo, 2012).

Diez meses después de esta resonante inauguración, Deolinda del Pilar Buenuleo - de la comunidad Buenuleo e integrante del Directorio de Wall Kintun TV- denunció la falta de apoyo institucional en la Radio Kalewche FM de Esquel (2013). Allí expresó que el canal había surgido por iniciativa 
de Diego Pereyra Cotaro, consejero por la Zona Andina del Consejo de Desarrollo de Comunidades Indigenas, quien expresó el deseo del gobierno nacional de impulsar un canal de televisión en esa comunidad. Dos semanas antes del 7 de diciembre de 2012, notificaron sobre la adjudicación de la licencia, por lo que Cotaro les anunció el nombre del canal y su modo de organización. Buenuleo denunció a Diego Cotaro, Matías Melillán -integrante del INAI- y Carlos Catrileo -integrante de la Coordinadora de Comunicación Audiovisual Indígena- por la utilización política de esta iniciativa, ya que la comunidad mapuche solo había puesto la firma para efectivizar la licencia, y también por promesas de capacitación, sueldos para los integrantes del canal, convenios con diferentes organismos y viajes a distintas provincias, que nunca se gestionaron.

En 2012, se realizó en Bariloche la VI Audiencia Pública por la Ley de Servicios de Comunicación Audiovisual, organizada por la Defensoría del Público de la Nación. En esa oportunidad, Buenuleo expresó:

¿Qué es Wall Kintun TV? Un transmisor de 600 que dio en comodato AFSCA, una PC con un playout también en comodato facilitado en este caso por AVC Video Cable y un espacio prestado por la provincia de Río Negro en el Centro Administrativo provincial. Es un grupo de gente que trabaja sin tener un sueldo, es un canal de televisión sin cámaras, sin máquinas para editar, es un espacio vacío (Comambiental, 2013, en linea).

En agosto de 2015 los integrantes de la comunidad ocuparon las instalaciones de AFSCA para reclamar el financiamiento del 10\% del presupuesto nacional y del presupuesto del INAI. Al respecto, expresaron: "No podemos vender publicidad porque el canal no se ve debido al transmisor de poco alcance que nos otorgaron" (Diario de Bariloche Noticiero AVC, 10/08/2015, en línea).

En octubre de ese mismo año, según informa el portal de la Agencia Digital de Noticias de Río Negro, "el Sindicato de Trabajadores de Prensa de Bariloche y Zona Andina señaló que "el canal Wall Kintun TV nació en 2012 como la primera emisora del país gestionada por una comunidad indígena. Tres años después agoniza jaqueada por el ahogo económico y las promesas incumplidas" " (31/10/2015, en línea). En la misma nota denunciaba: «El noticiero del canal comenzó a emitir en abril gracias a un aporte del 
Fomeca (Fondo Concursable de Fomento para Medios de Comunicación Audiovisual) que venció este mes y no fue renovado. Hace pocos días el programa dejó de salir al aire porque se agotó ese fondo, del que salían la magra remuneración de los seis periodistas y técnicos del canal y los gastos de movilidad para producir los contenidos (Agencia Digital de Noticias de Río Negro, 31/10/2015, en línea).

En declaraciones a Señales, una agencia de noticias de Bariloche, Martín Costa, delegado de AFSCA en dicha localidad, informó:

Apoyamos con numerosos recursos estos proyectos, porque queremos acompañar a las comunidades que decidieron organizarse $\mathrm{y}$ tener sus propios medios con identidad cultural. Reitero: recibieron un transmisor en comodato, numerosas capacitaciones, casi $900 \mathrm{mil}$ pesos ya cobrados, alrededor de 80 mil más este mes, otros $340 \mathrm{mil}$ si el directorio confirma lo que ya está preaprobado y están en condiciones de concursar en 15 días por otros 2 millones más de pesos $(10 / 11 / 2015$, en línea).

Y destacó: "En estos últimos tres años, AFSCA cumplió con el 100\% de sus responsabilidades. Lo que ocurre es que la ley prevé que el Estado acompañe con recursos el financiamiento de estos medios, no que los mantenga en su totalidad" (Señales, 10/11/2015, en línea).

Al día siguiente, en el mismo blog se informó que esta situación se había regularizado el día anterior, cuando se realizó una reunión entre autoridades de AFSCA e integrantes del canal. Como resultado, se suscribió un acta en la que el AFSCA se comprometió a garantizar la participación de los pueblos originarios respecto al proyecto de ley en donde se resuelve el presupuesto anual de la LSCA y acompañar a Wall Kintun TV en la participación de próximas líneas del FOMECA y en capacitaciones al personal del canal. Además, los integrantes del canal, ante los dichos de Costa, solicitaron que AFSCA corrija la información sobre los subsidios del FOMECA obtenidos por la comunidad (Señales, 10/11/2015).

En abril de 2016, los integrantes del canal Visión Indígena publicaron un video en YouTube en el que comunicaban que durante 2014 el canal había sobrevivido gracias a unas becas de $\$ 750$ que les había otorgado el Ministerio de Trabajo y a capacitaciones organizadas por el AFSCA y por la Defensoría del 
Público. Allí, explicaron que la programación del canal comenzaba diariamente a las $14: 30$ y a las 19 se emitía el informativo diario, que se repetía al día siguiente a las 13. El contenido era documentales que daban a conocer la lucha de los pueblos originarios, las costumbres, el idioma y programas de interés general, así como también musicales del canal Encuentro. Oscar Romero, integrante del canal, expresaba: "El objetivo es que la sociedad comprenda que hay otras formas de ver, entender y vincularse con el mundo, que la cultura del pueblo mapuche tiene mucho para decir" (Visión Indígena, 11/04/2016, en línea).

Aquí, también, Deolinda explicó que el transmisor de aire tiene un alcance de 15 cuadras de donde se encuentra ubicado el estudio (aunque se ha podido visualizar en áreas más lejanas), y que también se encuentran en el canal 10 de cable de AVC Bariloche. Deolinda afirmó: "El canal no llega a las comunidades porque el alcance de aire es bajo, sólo se puede visualizar si las comunidades tienen cable" (Visión Indígena, 11/04/2016, en línea).

Posteriormente, se anunció que continuaban a la espera de los fondos de Nación y que sus integrantes, "sin salario y sin seguridad social, siguen firmes en su propósito de sacar adelante el canal" (Visión Indígena, 11/04/2016, en

línea). En octubre de 2015, el canal debió suspender la emisión del noticiero Muley Zungu porque se venció el tiempo de aplicación de los aportes del FOMECA y no logró que se renovaran.

Por último, cabe destacar que dos días antes de su inauguración se lanzó una propuesta para que el canal fuera financiado por la sociedad civil a través de una página en internet mediante la cual se podía colaborar. En el mencionado sitio web, los productores del canal expresan: "Necesitamos completar el equipamiento de edición y play out» (Idearme, en línea).

\section{Reflexiones finales}

Este recorrido intentó exponer cómo la "letra" de la LSCA construyó un nuevo paradigma legal latinoamericano de los servicios de comunicación en relación con el interés público, entendido como esencial para el bienestar de la sociedad y de sus miembros (McQuail, 2010). Esta perspectiva, que implica reconocer el papel no solamente económico sino sociocultural de los sistemas 
de medios, ${ }^{3}$ no pudo ser abordada en la experiencia que es objeto de este trabajo, existiendo un gran distanciamiento entre lo consignado en la ley y las medidas implementadas. Basta observar el incumplimiento de los artículos en cuanto al financiamiento específico reconocido para los medios de comunicación de pueblos originarios y el apoyo a través de los FOMECA (en el que compiten con otras organizaciones perdiendo de esta manera la categoria jurídica especial que los reconoce como pueblos dentro del Estado Nacional). Si bien la normativa establece diferentes tipos de financiamiento para los medios de pueblos originarios, que permitan garantizar la pluralidad de la propiedad y de los contenidos, el Estado debe facilitar el mínimo tecnológico necesario para operar. La conformación monopólica y mercadocéntrica del sistema de medios impide el ingreso de las minorias sociales, y aquí el derecho a comunicar no puede quedar supeditado al de la comunicación como mercancía. El concepto de universalidad implica la disposición de una pluralidad de contenidos, incluso de aquellos que menos rentabilidad ocasionan, por lo que el Estado debe garantizar el apoyo económico.

Tampoco se recupera el reclamo inicial de los pueblos originarios de ser "sujetos de comunicación" y el espíritu de la LSCA de ingresar a nuevos agentes sociales como propietarios y como productores de contenidos. Los objetivos de acceso, de participación y de diversidad cultural ${ }^{4}$ quedan en la letra de la ley y las críticas al etnocentrismo en las aspiraciones de un nuevo ordenamiento por parte de las comunidades originarias. Considerar a los pueblos originarios como sujetos de emisión de contenidos implica asegurarles a estos agentes sociales la posibilidad de que ocupen el espacio de los medios con sus estéticas y sus narrativas.

La utilización política denunciada por los integrantes del canal remite a la importancia de pensar la responsabilidad del Estado para la creación de medios no gubernamentales y no estatales con el objeto de reivindicar el derecho a la comunicación de todos los ciudadanos y las ciudadanas. Si se cambiaran estas prácticas la ciudadanía podría empezar a reconocer la importancia de contar con medios públicos de comunicación. 
Cabe destacar que si bien esta experiencia no logró adecuarse en su totalidad al espíritu de la normativa, si facilitó el reconocimiento de los derechos de los pueblos originarios como sujetos de comunicación lo que resulta en el empoderamiento de la comunidad Buenuleo para reclamar y, como ellos lo afirman, "seguir firmes con el propósito de sacar adelante el canal».

Finalmente, se plantea un desafio: ¿cómo garantizar el derecho a la comunicación de los pueblos originarios sin hegemonizar sus prácticas y sus cosmovisiones específicas?

\section{Referencias}

Agencia Digital de Noticias Río Negro (31 de octubre de 2015). Canal Wall Kintun, ahogo económico y desaparición.

Recuperado de https: / /www.adnrionegro.com.ar/2015/10/canal-wall-kintunahogo-economico-y-desaparicion /

Badenes, D. y González, N (2014). La lucha por la democracia comunicacional en Argentina. En M. Lozano y J. Flores (Coords.), Democracia y sociedad en la Argentina contemporánea. Reflexiones sobre tres décadas. Bernal, Argentina: Universidad Nacional de Quilmes.

Badillo, Á.; Mastrini, G. y Marenghi, P. (2015). Teoria crítica, izquierda y políticas públicas de comunicación: el caso de América Latina y los gobiernos progresistas. Comunicación y Sociedad, (24), 95-126. Recuperado de https: / / www.redalyc.org/ articulo.oa?id=34639625005

Becerra, M. y Mastrini, G. (2009). El problema de la concentración (Documento de cátedra). Introducción a la economía política de la comunicación. Bernal, Argentina: Universidad Nacional de Quilmes. 
Coalición por una Radiodifusión Democrática (2004). 21 puntos básicos por el derecho a la comunicación. Telam. Recuperado de http://www.telam.com.ar/advf/imagenes/especiales/docume ntos/2012/11/509435587ec92.pdf

Comambiental (2 de noviembre de 2013). El primer canal indigena busca descolonizarse. Recuperado de http:/ /www.comambiental.com.ar/2013/11/el-primer-canalindigena-busca.html

Diario de Bariloche Noticiero AVC (10 de agosto de 2015). Wall Kintun TV reclama que se cumpla la Ley de Medios [Archivo de video]. Recuperado de https:/ / www.youtube.com/watch?v=QZriZY9WtBI

García Canclini, N. (2004). Modelos latinoamericanos de integración y desintegración. En N. García Canclini, Diferentes, desiguales y desconectados (pp. 131-145). Barcelona, España: Gedisa.

Gutiérrez Ríos, F. (2014). We Aukiñ Zugu. Historia de los medios de comunicación mapuche. Santiago de Chile, Chile: Quimantú.

Ideame (2012). Wall kintun en toda Bariloche [Archivo de video]. Recuperado de

https: / www.idea.me/proyectos / 566/wall-kintun-en-todabariloche 
Kalewche FM (4 de noviembre de 2013). Bariloche: la comunidad mapuche Buenuleo denunció el vaciamiento de Wall Kintun TV. Recuperado de http:/ / www.kalewchefm.org/noticias/la-tijereta/2264bariloche-la-comunidad-mapuche-buenuleo-denuncio-elvaciamiento-de-wall-kintun-tv

Ley Nacional 26.522 de Servicios de Comunicación Audiovisual (2009). Recuperado de http:/ / servicios.infoleg.gob.ar/infolegInternet/anexos / 155000 $-159999 / 158649 /$ norma.htm

Lorenzo, D. (5 de noviembre de 2012). El 7D saldrá al aire en Bariloche Wall Kintun, la TV mapuche. Uno Entre Ríos. Recuperado de https://www.unoentrerios.com.ar/pais/7dsaldra-al-aire-bariloche-wall-kintun-la-tv-mapuchen896515.html

Manasanch, A. (2010). Pueblos Originarios: comunicación con identidad. Trampas de la Comunicación y la Cultura, (69), 7681. Recuperado de http:/ / sedici.unlp.edu.ar/bitstream/handle/10915/35582/D ocumento_completo.pdf?sequence $=1$

Marino, S.; Mastrini, G; Becerra, M.; Rubini, C. y Espada, A. (2015). Diagnóstico sobre el acceso del sector sin fines de lucro a medios audiovisuales en la Argentina. Licencias, autorizaciones, permisos y fondos concursables. Recuperado de https://n9.cl/zjp7

Mastrini, G. y Aguerre, C. (2007). Muchos problemas para pocas voces. La regulación de la comunicación en el siglo XXI. Diálogo Político, XXIV(3). 
McQuail, D. (2010). La regulación de los medios (trad. Carla Rodríguez Miranda y Ana Bizberge). Leicester, Inglaterra:

University of Leicester, Mimeo. Recuperado de http:/ / politicasyplanificacion.sociales.uba.ar/wpcontent/uploads/sites/121/2014/07/mcquail_la_regulacion_ de_los_medios.pdf

Premici, S. (8 de diciembre de 2012). Una televisión originaria. Página/ 12. Recuperado de https:/ /www.pagina12.com.ar/diario/elpais/1-209476-201212-08.html

Señales (10 de noviembre de 2015). La AFSCA defendió su gestión ante Wall Kintun TV. Recuperado de https://seniales.blogspot.com/2015/11/1a-afsca-defendio-sugestion-ante-wall.html

Señales (11 de noviembre de 2015). Wall Kintun TV: Terminó la toma y fijan las falacias de un comunicado de la AFSCA. Recuperado de https://seniales.blogspot.com/2015/11/wallkintun-tv-levantaron-la-toma-y.html

Tremblay, G. (2005). El Informe MacBride, siempre de actualidad. Quaderns del CAC, (21), 21-22. Recuperado de https: / / www.cac.cat/sites / default/ files / 2019 05/Q21_tremblay_ES.pdf

Visión Indígena (14 de diciembre 2012). Visión Indígena: Ley de Servicios de Comunicación Audiovisual (2 de 3) [Archivo de video]. Recuperado de https://www.youtube.com/watch?v=Trx-x1siJ-I

Visión Indígena (11 de abril de 2016). Denuncia de integrantes del Canal "Wall Kintun TV" [Archivo de video]. Recuperado de https:/ / www.youtube.com/watch?v=Gkkz8dz-IQQ 
Yanniello, F. (2014). Descolonizando la palabra: los medios de comunicación del pueblo Mapuche en Puelmapu. La Plata, Argentina: Ediciones de la Caracola.

\section{Notas}

1 Estas categorias se definen retomando a Inés Pousadela (2010), quien conceptualiza las experiencias de izquierda, de centroizquierda y progresistas en la región latinoamericana como alternativas al modelo neoliberal en el contexto de la globalización (en Badillo, Mastrini \& Marenghi, 2015).

2 Guillermo Mastrini y Carolina Aguerre (2007) proponen repensar el papel activo del Estado a partir de la reregulación, ya que el Estado intervino con una racionalidad económica y apoyó procesos de acumulación de capital en detrimento de las lógicas culturales.

3 "... ya que los mismos juegan un rol esencial en la expresión y continuidad de la identidad nacional y cultural; reflejan la diversidad regional, étnica y otras formas de diversidad; y cohesionan -a través de la intercomunicacióna la sociedad como un todo así como a las comunidades particulares y sus elementos constitutivos" (McQuail, 2010, p. 4).

4 Entendida como la posibilidad de representación de otras maneras de interpretar el mundo, teniendo en cuenta el rol preponderante de los medios de comunicación para fijar y para legitimar pautas de conducta y modos de ser y de hacer colectivos. 